

\title{
Estimating effects of removing negative features on engineering analysis
}

\author{
Ming Li and Shuming Gao* \\ State Key Laboratory of CADESCG, Zhejiang University, China P.R. \\ Ralph R. Martin \\ School of Computer Science $\mathcal{G}$ Informatics, Cardiff University, UK
}

\begin{abstract}
This paper provides a general framework for the quantitative estimation of the effects of removing negative features on engineering analysis, or modification sensitivity for short. There are two main applications: (i) when defeaturing models so that finite element analysis may be carried out more quickly and with lower memory requirements, and (ii) when performing iterative design based on finite element analysis. Our approach can handle large as well as small features, and features with Neumann/natural boundary conditions prescribed on them; previous methods have difficulties in handling such cases.

Estimation of the modification sensitivity is achieved by reformulating it as a modeling error caused by use of different mathematical models to describe the same engineering analysis problem. Results are obtained using the dual weighted residual (DWR) method in combination with a heuristic assumption of small variation of dual solution after defeaturing. The final derived sensitivity estimator is expressed in terms of the difference of local boundary integrations over the feature boundary, which can be explicitly evaluated using solutions defined on the defeatured model.

The algorithm's performance is demonstrated using a Poisson equation. Comparisons to results obtained by previous approaches indicate it is both accurate and computationally efficient.
\end{abstract}

Key words: modification sensitivity, design sensitivity, engineering analysis error, large design changes, DWR.

\section{Introduction}

Computational simulations, or engineering analysis, predict the physical behavior of a designed engineering component under various boundary constraints and external loadings. They allow engineers to investigate, visualize and test various physical and mechanical properties and operations on a designed object before constructing a real physical prototype, shortening the design cycle. Engineering analysis is generally performed using finite element (FE) analysis on a mesh derived by discretizing a CAD model.

Such simulation-based design typically involves a process of repeated design changes which modify a designed model by adding or removing design features, or adjusting geometric parameters of features, to meet ultimate design objectives of functionality, manufacturability, aesthetics, and so on. A design objective is typically measured using local quantities of engineering interest [16], such as pointwise displacements or average temperatures over a region

\footnotetext{
*smgao@cad.zju.edu.cn
}

etc; global values are not able to provide specific enough information about the model. Each time a design change is made, for example, adding a feature to the model, the engineering analysis problem must be solved again for the modified model in order to obtain these target local quantities. See Fig. 1(a). Such repeated analyses can be very expensive if the model's geometry is complex or a sophisticated or accurate analysis is required.

The overall computational efficiency can be much improved if the previous computational results for the prior design stage can be reutilized for the modified model. This idea can be put into practice by estimating differences between local quantities in the prior and current design, allowing decisions on design changes to be made. See Fig. 1(b). In some cases these changes may be small and local, but in other cases, relatively large features may be added to or removed from a model. The approach reported here can handle cases where large negative features (with prescribed Neumann or natural boundary conditions) are removedprevious work has considered smaller, more local shape changes. (A companion paper also considers the case of pos- 


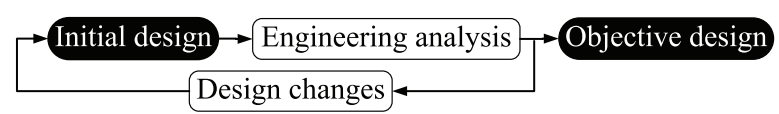

(a)

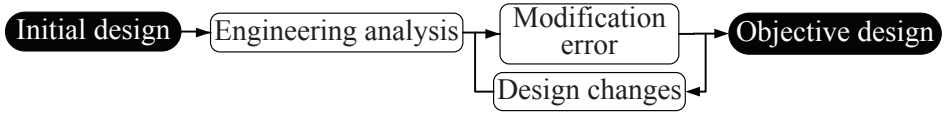

(b)

Fig. 1. Different strategies for simulation-based design. (a) repeated engineering analysis is required for each design change; (b) using modification sensitivity estimation, only one step of engineering analysis is required for all model modifications.

itive features, but these require rather more heuristic methods [20]; this paper provides a sounder and more theoretical background to the approach). In summary, we consider how to estimate the effects of removing large design features on results of engineering analysis, measured in terms of changes in a particular quantity of engineering interest, in other words, the modification sensitivity for that quantity and model.

The problem of modification sensitivity estimation also arises when performing model simplification for analysis. Here, the aim is to remove design features that have little impact on the results of analysis, allowing the analysis to be performed more quickly on a simpler model. Irrelevant geometric details can significantly increase the time and computational complexity both for the meshing process and the FE analysis performed on it [29,19]. Worse, they may even lead to mesh generation failure [29] or illconditioned computations [24] that may produce inaccurate analysis results. In extreme cases, the fully-featured problem may be too complex for an engineering analysis to be tractable, and model simplification is essential in such cases. Consider, for example, Fig. 2. Finite element analysis requires both geometric adaptivity and smooth mesh size transitions, leading to a mesh with far more elements for the fully-featured model than for a corresponding defeatured model. The benefits of suppressing features come at a cost of somewhat different results of the analysis. Understanding such modification sensitivity is essential to ensure that a desired analysis accuracy can still be met after defeaturing.

As noted, this paper focuses on the modification sensitivity estimation of the case of removing negative features (of Neumann/natural boundary conditions) within the model's interior or along its boundary. This problem is of particular relevance in plate or shell analysis [8], where design changes are usually made within the material of the model. Estimating sensitivity to the creation of such negative features is also important in classical topological optimization [10], although that topic focuses on negative features of infinitesimal size while this paper focuses on negative features of possibly large size, which can be in the model's interior or on its boundary.

The general framework provided in this paper for estimating the effects of removing negative features on engineering analysis is based on reformulating the modification sensitivity, originally caused by a geometric difference, as a modeling error, caused by the use of different PDEs to mathematically model a physical phenomenon over the same geometric model. Estimating this modeling error is achieved using the dual weighted residual (DWR) method, originally developed in $[3,22]$, in combination with a heuristic assumption that the difference between the dual solutions of the fully-featured model and the defeatured model is small. This results in the derived modification sensitivity being expressed in the form of a local integration over the feature's boundary, which can be explicitly evaluated using engineering analysis results from the defeatured model.

The remainder of the paper is organized as follows. Related work is discussed in Section 2. The problem studied in this paper is defined more precisely in Section 3. Estimating the modification sensitivity using DWR is described in Section 4. The computational efficiency and accuracy of our approach and related approaches are discussed in Section 5, while numerical experiments are presented in Section 6. Conclusions are drawn in Section 7.

\section{Related work}

Unlike the well-studied analysis error estimates for FE approximation [17], the problem of estimating modification sensitivity is rarely studied, and was stated to be an open problem in a recent survey on physically-based model simplification [26]. Previous research work closely related to this topic includes studies on design sensitivity, feature sensitivity, and reanalysis techniques.

Design sensitivity analysis computes the derivatives of model response quantities with respect to design variables, and plays a critical role in design optimization. Given sufficiently small model modifications, shape sensitivity analysis [6] allows designers to compute the change in local quantities of interest when a model boundary is perturbed, based on infinitesimal changes, while topological sensitivity analysis $[10,25]$ computes the change when an infinitesimal circular hole is created within an existing geometry. Extensions of topological sensitivity analysis to arbitrarilyshaped features are given in $[2,21]$. Explicit analytical expressions have also been derived for, e.g. the case of a classical Poisson equation [11]. Recently, a practical, efficient numerical approach was proposed by Suresh et al $[15,27,28]$ for estimating the topological sensitivity (called feature sensitivity there) to creation of an arbitrarily-shaped small internal hole. However, design sensitivity analysis and its variants are essentially dependent on asymptotic expansions of target functionals, which strongly limits the nature of permissible sizes and locations of the features involved. They are hard to apply directly to the problem of large design changes we consider here.

Our sensitivity estimator is achieved by extending the DWR method. The DWR method was originally developed 


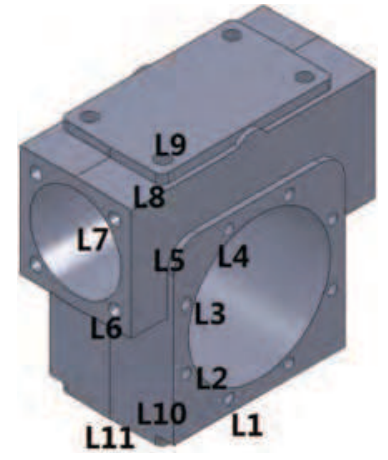

(a) Original CAD model

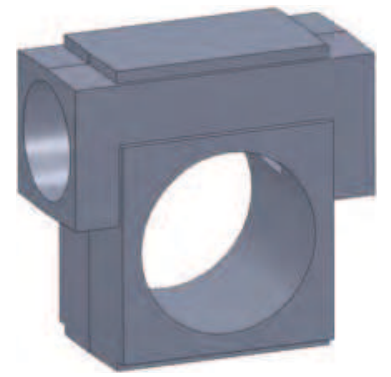

(b) Defeatured CAD model

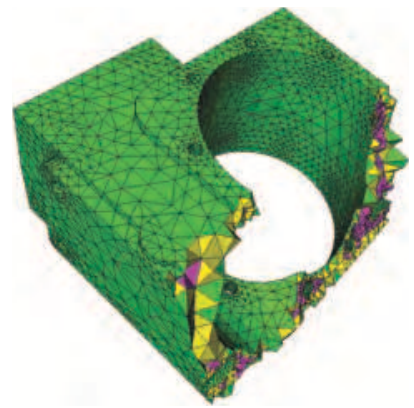

(c) Mesh for original model with 86030 elements

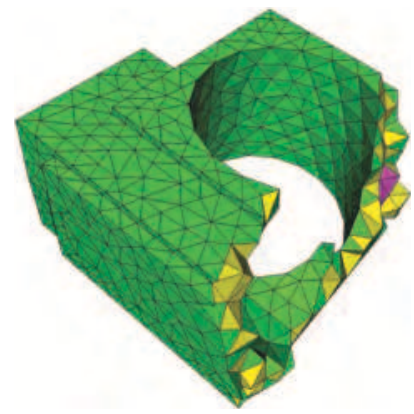

(d) Mesh for defeatured model with 3990 elements

Fig. 2. Suppressing features (36 holes, 8 blends and 2 extrusions) from the fully-featured model in (a) leaves the defeatured model in (b). It needs fewer mesh elements to represent it to the desired accuracy.

by Becker and Rannacher [3,4] to estimate goal-oriented FE approximation error for a broad class of nonlinear problems. It was then extended by Oden and Prudhomme [22] to modeling error estimation. This prior work only considers problems in which the underlying geometry over which analysis is performed, and the associated boundary conditions, remain unchanged before and after approximation. Recently, Cnossen considered [7] the possibility of applying DWR to fluid-flow problems where varying boundary conditions may be used with fixed geometry. We go further and consider utilizing DWR for quite general geometric design changes, extending this previous work.

All the above mentioned approaches consider goaloriented modification sensitivity estimation. There are also heuristic approaches studying modification sensitivity in terms of global energy differences [13]. However, an essential starting step is that a cut boundary has to be selected around the suppressed feature to allow for local computation, and there is no obvious simple approach for doing so.

Another approach for estimating modification sensitivity is based on reanalysis techniques $[8,18]$, which links small geometric modifications of a model to variations in its associated stiffness matrix. However, modifying large design features may drastically change this matrix. Moreover, for successful implementation of reanalysis techniques, steps of model preparation, meshing and nodal mapping are required, which are non-trivial problems in their own right.

\section{Problem statement}

We now further explain and define the problem of estimating effects of removing negative features on engineering analysis, i.e. modification sensitivity.

In our problem, we assume that we have a general engineering analysis problem defined over two different models, which for simplicity we call the fully-featured model and a defeatured model. The engineering analysis problem concerning, e.g. heat transfer or elasticity analysis, is generally described by one or more partial differential equations (PDEs) plus certain prescribed boundary conditions, e.g. external loadings or fixed temperatures. Performing engi- neering analysis on a model requires computing the field solutions of the corresponding boundary value PDE problem over the model, typically using FE methods.

The fully-featured model might be a model before feature suppression when performing model simplification, or it might be a modified model with one or more added negative features in a simulation-based design scenario. It typically has a complex geometry and its PDE solutions are very hard to compute or even intractable. The defeatured model might be a simplified model after removing features, or in a design scenario, a simpler model before one or more negative features have been added to the design. Typically it has much simpler geometry and its PDE solutions are much easier to determine (or may even be provided). Since only negative features are considered here, the fullyfeatured model is contained within the volume of the defeatured model.

The negative features to be suppressed are assumed to be given in advance. This paper mainly focuses on estimating the effects of removing such a single negative feature on engineering analysis. A general approach to utilizing the results to help simplify complex models is not a trivial task and will be addressed in our future work.

Geometric differences between the fully-featured model and the defeatured model perturb the underlying solutions to the analysis problems defined over these models, and affects values of the local quantities of interest. Our objective is to quantitatively estimate the modification sensitivity in terms of changes of local quantities of interest due to the geometric difference between the fully-featured model and the defeatured model; we wish to do so without explicitly solving the analysis problem for the fully-featured model.

A mathematical formulation is now given. The problem is first analysed for the case of removing a single negative feature; its extension to multiple features will be discussed at the end of this section. As illustrated in Fig. 3, we have a fully-featured model $\Omega-\omega$ and a defeatured model $\Omega$, where $\omega \subset \Omega$ is a negative feature to be suppressed, provided by the user or other software. The engineering analysis problem over $\Omega$ or $\Omega-\omega$ is defined as follows. Firstly, we define the solution $u$ over $\Omega-\omega$ to be the solution to 


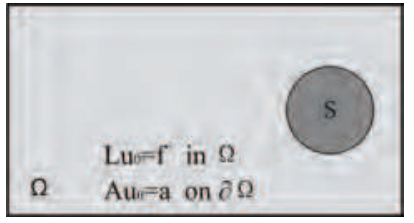

(a) Defeatured model $\Omega$

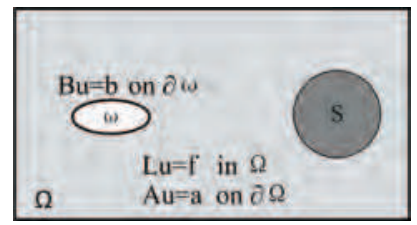

(b) Fully-featured model $\Omega-\omega$
Fig. 3. Engineering analysis problems for fully-featured and defeatured geometric models.

the following PDE problem:

$$
\left\{\begin{array}{l}
L u=f \text { in } \Omega-\omega \\
A u=a \text { on } \partial \Omega \\
B u=b \text { on } \partial \omega
\end{array}\right.
$$

where $\partial \Omega$ or $\partial \omega$ denotes the boundary of $\Omega$ or $\omega, L, A, B$ are abstract linear or nonlinear differential operators governing the physical phenomenon within the model interior, bounded by $\partial \Omega$ and $\partial \omega$. The problem and boundary conditions are further characterised by $f, a$ and $b$. For the particular problem studied in this paper, Neumann or homogeneous Dirichlet boundary conditions are assumed over boundary $\partial \Omega$, and Neumann boundary conditions are assumed over feature boundary $\partial \omega$ in this paper.

Suppressing feature $\omega$ from model $\Omega-\omega$, leads to a new PDE problem with solution $u_{0}$ satisfying

$$
\left\{\begin{array}{l}
L u_{0}=f \text { in } \Omega, \\
A u_{0}=a \text { on } \partial \Omega .
\end{array}\right.
$$

Note that the boundary conditions prescribed over feature $\omega$ in Eq. (1) disappear, due the removal of feature $\omega$.

Typically, the analyst wishes to know certain results of the analysis such as average local stresses or temperatures. Such quantities of engineering interest may be defined in terms of an integral over a local region $S \subset \Omega-\omega$ :

$$
Q(u)=\int_{S} l(u) d \Omega,
$$

where $q(\cdot)$ is a linear or nonlinear bounded functional defined over the solution space.

The geometric difference between $\Omega$ and $\Omega-\omega$ causes the corresponding solutions $u_{0}$ and $u$ to differ, and which in turn affects the quantities of interest $Q\left(u_{0}\right)$ and $Q(u)$. The estimated effect on the engineering analysis results of removing a design feature, i.e. the modification sensitivity, is then given by

$$
e(\Omega, \Omega-\omega)=Q(u)-Q\left(u_{0}\right) .
$$

We assume the solution $u_{0}$ is known, and we wish to avoid explicitly computing $u$.

We now consider what happens for a model containing multiple features, and how to determine the modification sensitivity when a single feature or multiple features are removed. The ideas are simplest explained in terms of two features, but generalise to multiple features. Suppose model $M=\Omega-\omega_{1}-\omega_{2}$ is a complex fully-featured model containing two negative features $\omega_{1}, \omega_{2}$. These two features can always be assumed to be disjoint in their interior, as we can replace $\omega_{1}$ with $\omega_{1}-\left(\omega_{1} \cap \omega_{2}\right)$ if they are not. The effect on the quantity of interest due to interactions between them has to be considered in the cases of multiple features. If $\omega_{1}$ and $\omega_{2}$ are not physically dependent (normally meaning they are not geometrically intersecting or very close), the two features can be processed separately to estimate their associated modification sensitivities. Furthermore, for each feature, say $\omega_{1}$, two different sensitivity estimation calculations can be performed (i) taking the fully-featured and defeatured models as $\Omega-\omega_{1}$ and $\Omega$, that is the sensitivity is computed as $e\left(\Omega, \Omega-\omega_{1}\right)$, and (ii) taking them as $\left(\Omega-\omega_{2}\right)-\omega_{1}$ and $\Omega-\omega_{2}$, that is the sensitivity is computed as $e\left(\Omega-\omega_{2},\left(\Omega-\omega_{2}\right)-\omega_{1}\right)$. If there are no feature interactions, the two calculations produce the same result. Note, however, that the former strategy involves the same defeatured model $\Omega$ for different features $\omega_{1}$ and $\omega_{2}$ and has a defeatured model of greater geometric simplicity. For a complex engineering part with hundreds of features, the former will be much cheaper computationally, so is the strategy used in our experimental implementations in Section 6. On the other hand, if $\omega_{1}$ and $\omega_{2}$ are physically dependent, they should be taken to be a single high-level feature composed of multiple sub-features, and a corresponding modification sensitivity estimate computed.

\section{Modification sensitivity estimation using DWR}

Estimation of the modification sensitivity defined by Eq. (4) is ultimately achieved by reformulating it as the modeling error between two different weak formulations (which we explain shortly). The modeling error is then estimated using the dual weighted residual (DWR) method [22]. We first explain the concept of weak formulations and the DWR method. Then the concrete approach of reformulating the modification sensitivity as a modeling error is described. Finally, our modification sensitivity estimator is derived.

\subsection{Weak formulations}

Weak formulations are an important tool to permit the transfer of concepts from linear algebra to solving PDEs under certain boundary conditions. They provide a uniform language for describing various engineering analysis problems, and are the base of FE analysis.

Taking a concrete example, we use the following Poisson equation to illustrate the derivation of a weak formulation and its properties. Suppose we wish to find a solution $u$ to the problem:

$$
\begin{cases}-\operatorname{div}(\nabla u)=f & \text { in } \Omega, \\ u=u_{d} & \text { on } \Gamma_{D} \\ \nabla u \cdot n=h & \text { on } \Gamma_{N}\end{cases}
$$


where $\Gamma_{D}, \Gamma_{N}$ are Dirichlet and Neumann boundaries, which taken together make up $\partial \Omega ; n$ is a unit outward normal vector to $\Gamma_{N}$.

In the general framework of finite element analysis, the weak form of Eq. (5) can be obtained by multiplying by a test function $v$ on the left and right hand sides, and then performing integration by parts, leading to a new problem: find $u \in V$ such that

$$
\int_{\Omega} \nabla u \cdot \nabla v d \Omega=\int_{\Omega} f v d \Omega+\int_{\Gamma_{N}} h v d \Gamma, \quad v \in V_{0},
$$

where the solution space $V$ is defined as

$$
V=\left\{v \in H^{1}(\Omega): v=u_{d} \text { on } \Gamma_{D}\right\},
$$

and the test space $V_{0}$ is defined as

$$
V_{0}=\left\{v \in H^{1}(\Omega): v=0 \text { on } \Gamma_{D}\right\}
$$

for a Hilbert space $H^{1}(\Omega)$ over region $\Omega$.

Note that the Dirichlet boundary condition is built into the definition of the solution space $V$, while functions in the test space $V_{0}$ are zero on the Dirichlet portion of the boundary. This is in contrast to the Neumann case where the solution and the test functions are unrestricted on the boundary.

\subsection{DWR in modeling error estimation}

We now introduce the use of DWR for modeling error estimation. Following common practice, the convention $N(\cdot ; \cdot)$ is used to indicate a function linear in all arguments that follow the semicolon but possibly nonlinear for preceding arguments.

The problem of estimating modeling error is defined for the following two abstract nonlinear problems expressed in weak form: find solutions $u, u_{0}$ such that

$$
N(u ; v)=F(v) \quad \forall v \in V,
$$

and

$$
N_{0}\left(u_{0} ; v\right)=F_{0}(v) \quad \forall v \in V,
$$

where $N(\cdot ; \cdot), N_{0}(\cdot ; \cdot)$ are semilinear forms defined on a Banach space $V$, and $F(\cdot), F_{0}(\cdot)$ are linear functionals on $V$. We assume that each equation admits a unique solution for either $u$ or $u_{0}$.

Suppose that for the above two different modeling equations, solution $u_{0}$ provides an approximation to the solution $u$. For a particular quantity of interest $Q(u)$ as in Eq. (3), the problem of modeling error estimation is to evaluate $Q(u)-Q\left(u_{0}\right)$ without explicitly solving $u$ from Eq. (7).

To do so, the formulation of an auxiliary problem, we consider an adjoint problem with respect to the prime problem, following [16]. The prime problem in Eq. (7) has the adjoint problem: find solution $p \in V$ such that

$$
N^{\prime}(u ; q, p)=Q^{\prime}(u ; q) \quad \forall q \in V,
$$

where $N^{\prime}(u ; q, p)$ and $Q^{\prime}(u ; q)$ are Gâteaux derivatives [22]. The corresponding adjoint problem for Eq.(8) is: find solution $p_{0} \in V$ such that

$$
N_{0}^{\prime}\left(u_{0} ; q, p_{0}\right)=Q^{\prime}\left(u_{0} ; q\right) \quad \forall q \in V .
$$

Defining the residual functional to characterize the degree to which $u_{0}$ fails to satisfy the problem in Eq. (7) as

$$
\mathcal{R}\left(u_{0} ; v\right)=F(v)-N\left(u_{0} ; v\right), v \in V,
$$

the following theorem is given in [22] for estimating the modeling error by dropping higher order remainder terms. Theorem 1 [22] When $u-u_{0}, p-p_{0}$ are small, any approximation $\left(u_{0}, p_{0}\right)$ of the solutions $(u, p)$ of $E q$. (9) gives an a posteriori error estimate of

$$
Q(u)-Q\left(u_{0}\right) \approx \mathcal{R}\left(u_{0} ; p_{0}\right)+\mathcal{R}\left(u_{0} ; p-p_{0}\right) .
$$

\subsection{Conversion from modification sensitivity to modeling error}

Based on the result in Theorem 1, we now give a general framework for modification sensitivity estimation. Let $u$, $u_{0}$ to denote solutions of Eqs. (1) and (2) defined respectively on $\Omega-\omega$ and $\Omega$, and $p, p_{0}$ for their respective adjoint solutions defined as in Eqs. (9) and (10).

As the fully-featured model is contained in the defeatured model, that is $\Omega-\omega \subset \Omega$, the part of the solution $u_{0}$ defined over geometry $\Omega-\omega$ is well defined, and is denoted by

$$
\bar{u}_{0}=\left.u_{0}\right|_{\Omega-\omega} .
$$

The solution $\bar{u}_{0}$ can seen as the solution of another engineering analysis problem defined over model $\Omega-\omega$ with particular Neumann conditions prescribed over the internal boundary $\partial \omega$ : find $\bar{u}_{0}$ such that

$$
\left\{\begin{array}{l}
L \bar{u}_{0}=f \text { in } \Omega-\omega, \\
A \bar{u}_{0}=a \text { on } \partial \Omega, \\
\bar{B} \bar{u}_{0}=\bar{b} \text { on } \partial \omega,
\end{array}\right.
$$

where the last term $\bar{B} \bar{u}_{0}=\bar{b}$ in this case corresponds to

$$
\frac{\partial \bar{u}_{0}}{\partial n}=\frac{\partial u_{0}}{\partial n}
$$

Generally, the term $\bar{B} \bar{u}_{0}=\bar{b}$ is different from the last equation of Eq. (1), that is $B u=b$, and it is this difference which is essentially the source of the modification sensitivity studied in this paper.

Let the corresponding abstract weak forms for Eqs. (1) and (13) be expressed as follows: find solutions $u, u_{0} \in V$ such that

$$
N(u ; v)=F(v) \quad \forall v \in V
$$

and

$$
N_{0}\left(\bar{u}_{0} ; v\right)=F_{0}(v) \quad \forall v \in V,
$$

i.e., $\bar{u}_{0}$ and $u$ are solutions of two different modeling equations, Eqs. (15) and (16).

Now, it is clear from Eq. (14) that

$$
Q(u)-Q\left(u_{0}\right)=Q(u)-Q\left(\bar{u}_{0}\right) .
$$

The left hand side of the equation, $Q(u)-Q\left(u_{0}\right)$, is the modification sensitivity defined over two different geometries $\Omega, \Omega-\omega$ considered here. The right hand side of the 
equation, $Q(u)-Q\left(\bar{u}_{0}\right)$, is the modeling error defined over the same geometry $\Omega-\omega$ caused by two different weak formulations, that is Eqs. (15) and (16). Their equality allows us to use the result in Theorem 1 to convert from modification sensitivity estimation to modeling error estimation.

Note that when applying the DWR method to modeling error estimation, the solution and test spaces for Eqs. (9) and (10) have to be the same Banach space $V$. This is generally not satisfied for general boundary settings as explained in Section 4.1, but is satisfied for the particular boundary conditions stated in Eq. (1), that is, the solution and test spaces of Eqs. (1), (13) and their associated adjoint problems are the same. Thus, based on Theorem 1 and noticing that $\bar{u}_{0}=u_{0}$ over $\Omega-\omega$, the following lemma can be given for modification sensitivity estimation:

Lemma 1 The modification sensitivity in Eq. (4) is given as

$$
Q(u)-Q\left(u_{0}\right) \approx \mathcal{R}\left(u_{0} ; p\right),
$$

where $u_{0}$ is the solution to Eq. (2), and $p$ is the adjoint solution to Eq. (1).

\subsection{Modification sensitivity estimate}

In this section, the sensitivity expression in Eq. (18) is further simplified to ultimately give a computable modification sensitivity estimate in the form of local boundary integrations. This section uses the notation that, given two general vectors $w, v$, their inner product over a region $S$ is

$$
\left.(w, v)\right|_{S}=\int_{S} w \cdot v d V
$$

Let the general expression of performing integration by parts for $\left.(L w, v)\right|_{\Omega-\omega}$ be

$$
\left.(L w, v)\right|_{\Omega-\omega}=H_{\partial \Omega}(w, v)+h_{\partial \omega}(w, v)-\left.L^{*}(w, v)\right|_{\Omega-\omega},
$$

where $L$ is the abstract differential operator in Eq. (1), $H_{\partial \Omega}(w, v)$ and $h_{\partial \omega}(\cdot, \cdot), L^{*}$ are functionals defined over $V \times$ $V$ expressed in forms of integrations over boundaries $\partial \Omega$ and $\partial \omega$, and $\Omega-\omega$.

Now, consider replacing $w$ in Eq. (19) by the particular solution $u$ from Eq. (1). Evaluating the specific boundary conditions defined by $A u=a$ over the boundary $\partial \Omega$ for $H_{\partial \Omega}(w, v)$, we get a functional $\bar{H}_{\partial \Omega}(u, v)$. Similarly, evaluating boundary conditions $B u=b$ defined over $\partial \omega$ for $h_{\partial \omega}(w, v)$, we get another functional $\bar{h}_{\partial \omega}(u, v)$. This leads to the corresponding weak formulation of Eq. (1)

$$
\left.(L u, v)\right|_{\Omega-\omega}=\bar{H}_{\partial \Omega}(u, v)+\bar{h}_{\partial \omega}(u, v)-\left.L^{*}(u, v)\right|_{\Omega-\omega} .
$$

Using the above notation, the sensitivity estimation term $\mathcal{R}\left(u_{0} ; p\right)$ in Eq. (18) can now further simplified:

Theorem 2 The modification sensitivity defined in Eq. (4) can be expressed as a difference between two local boundary integrations over the feature boundary $\partial \omega$ :

$$
Q(u)-Q\left(u_{0}\right) \approx \mathcal{R}\left(u_{0} ; p\right)=\bar{h}_{\partial \omega}\left(u_{0}, p\right)-h_{\partial \omega}\left(u_{0}, p\right),
$$

where $h_{\partial \omega}\left(u_{0}, p\right), \bar{h}_{\partial \omega}\left(u_{0}, p\right)$ are defined as above.
Proof: See Appendix A.

This theorem states that the modification sensitivity is essentially due to the difference of the PDE solutions along the boundary $\partial \omega$ before and after removing feature $\omega$.

Compared to the expression based on volume integration in Eq. (18), the sensitivity expression in Eq. (21) is simpler. However, Eq. (21) still involves the solution $p$, which is defined over the geometry $\Omega-\omega$ and is assumed to be unavailable. In order to derive a computable sensitivity estimate, we further divide $p$ into $p_{0}$ and $\varepsilon_{0}=p-p_{0}$. From the linearity of $\mathcal{R}\left(u_{0} ; p\right)$, we have

$$
\begin{aligned}
Q(u)-Q\left(u_{0}\right) \approx & \bar{h}_{\partial \omega}\left(u_{0}, p_{0}\right)-h_{\partial \omega}\left(u_{0}, p_{0}\right)+ \\
& \left(\bar{h}_{\partial \omega}\left(u_{0}, \varepsilon_{0}\right)-h_{\partial \omega}\left(u_{0}, \varepsilon_{0}\right)\right) .
\end{aligned}
$$

The first term $\bar{h}_{\partial \omega}\left(u_{0}, p_{0}\right)-h_{\partial \omega}\left(u_{0}, p_{0}\right)$ can be directly evaluated from the prime and adjoint solutions $u_{0}, p_{0}$ defined over the defeatured model $\Omega$. The second term $\bar{h}_{\partial \omega}\left(u_{0}, \varepsilon_{0}\right)-$ $h_{\partial \omega}\left(u_{0}, \varepsilon_{0}\right)$ is however dependent on the solution adjoint error $\varepsilon_{0}=p-p_{0}$ or $p$ defined over the feature boundary $\partial \omega$, and is not available.

This unavailable term can be further approximated by its exterior solution [5], i.e. the solution to the same analysis problem defined over geometry $R^{n}-\omega(n$ is the dimensionality of the model being analysed), using the same boundary conditions as those for the solution $u_{0}$ over feature boundary $\partial \omega$. Alternatively, it can be bounded using values based on global energy norm estimates of the primal and adjoint problems [16]. The first estimation strategy requires solution of a further analysis problem, and has specific requirements on the size and location of the target feature. The second approach, on the other hand, involves complex computations.

For the particular problem of modification sensitivity estimation of large features, due to the locality of the adjoint problems in Eq. (9), in terms of its source term and boundary terms, the difference between solutions $p$ and $p_{0}$ is typically negligible compared with the term $\mathcal{R}\left(u_{0} ; p_{0}\right)$, and may be simply discarded. This is stated in the following corollary.

Corollary 1 The modification sensitivity defined by Eq. (4) can be approximated by the difference of local boundary integrations:

$$
Q(u)-Q\left(u_{0}\right) \approx \bar{h}_{\partial \omega}\left(u_{0}, p_{0}\right)-h_{\partial \omega}\left(u_{0}, p_{0}\right),
$$

where $u_{0}, p_{0}$ are prime and adjoint solutions of Eq. (2), and $h_{\partial \omega}\left(u_{0}, p_{0}\right), \bar{h}_{\partial \omega}\left(u_{0}, p_{0}\right)$ are boundary integration terms over the feature boundary $\partial \omega$ defined by Eqs. (19) and (20). The above corollary lacks rigorous proof of validity of dropping the term with respect to $R\left(u_{0}, p-p_{0}\right)$. We hope to verify this in our future work. Various examples, including those shown in Section 6, demonstrate the accuracy of Corollary 1 for modification sensitivity estimation.

In practical implementations, the solutions $u_{0}, p_{0}$ are derived using $\mathrm{FE}$ methods from a very dense mesh generated from the defeatured model $\Omega$ ignoring FE approximation 
error. Based on these values, the modification sensitivity can be easily evaluated based on Eq. (23).

\section{Computational efficiency and accuracy}

In this section, we use a classical Poisson equation to compare the computational efficiency and accuracy of the proposed approach with other methods: direct FE analysis, feature sensitivity [27] and topological sensitivity [11,25].

Poisson's equation is often encountered in heat and mass transfer theory, elasticity, and other areas of mechanics and physics. Let the fully-featured and defeatured models respectively be $\Omega-\omega$ and $\Omega$. The solution prime solution for the defeatured model, $u_{0}$, is given by Eq. (5) after replacing $u$ by $u_{0}$; let $p_{0}$ be the corresponding adjoint solution. For $\Omega-\omega$ we wish to find the solution $u$ such that

$$
\begin{cases}-\operatorname{div}(\nabla u)=f & \text { in } \Omega-\omega, \\ u=0 & \text { on } \Gamma_{D}, \\ \nabla u \cdot n=h & \text { on } \Gamma_{N}, \\ \nabla u \cdot n=g & \text { on } \omega,\end{cases}
$$

where $\Gamma_{D} \cup \Gamma_{N}=\partial \Omega$.

The equivalent weak form for Eq. (24): find $u \in V$ such that

$$
N(u, v)=F(v), v \in V
$$

where

$$
\begin{gathered}
N(u, v)=\int_{\Omega-\omega} \nabla u \cdot \nabla v d \Omega-\int_{\partial \Omega_{N}} h v d \Gamma-\int_{\partial \omega} g v d \Gamma \\
F(v)=\int_{\Omega-\omega} f v d \Omega
\end{gathered}
$$

and $V=\left\{v \in H^{1}(\Omega): v \mid \Gamma_{D}=0\right\}$.

In the following, we use $P_{C}$ for the center of the smallest ball that encloses the internal feature $\omega$, and

$$
\tilde{p}_{0}=\left.p_{0}\right|_{P_{C}}, \nabla \tilde{u}_{0}=\left.\nabla u_{0}\right|_{P_{C}}, \nabla \tilde{p}_{0}=\left.\nabla p_{0}\right|_{P_{C}} .
$$

To make a comparison with other approaches, we first summarize below related methods for estimating modification sensitivity, including direct FE analysis (FEA), topological sensitivity analysis (TSA) and feature sensitivity analysis (FSA).

(1) FE analysis. The difference between $Q(u)$ and $Q\left(u_{0}\right)$ is evaluated directly by computing the field solutions $u, u_{0}$ from Eqs. (1) and (2). We ignore discrete FE approximation errors, and use the resulting value as ground truth when comparing other approaches.

(2) Topological sensitivity analysis [2,11,25]. Explicit expressions are determined for the modification sensitivity for the cases of an internal circular or elliptic hole within an existing geometry.

For a circular hole $\omega$ of radius $\epsilon$,

$$
Q(u)=Q\left(u_{0}\right)+t(\epsilon) D_{T} Q,
$$

where

$$
t(\epsilon)=-2 \pi \epsilon ; \quad D_{T} Q=g \tilde{p}_{0}, \text { when } g \neq 0 ;
$$

Table 1

Comparison of computational requirements of FEA, TSA, FSA and our proposed approach for modification sensitivity estimation.

\begin{tabular}{|c|c|ccc|}
\hline & FE analysis & \multicolumn{3}{|c|}{ Integration } \\
\hline & Fine Coarse & Exterior & Volume & Boundary \\
\hline TSA & $\sqrt{ }$ & & & \\
\hline Ours & $\sqrt{ }$ & & & $\sqrt{ }$ \\
\hline FSA & $\sqrt{ }$ & $\sqrt{ }$ & & $\sqrt{ }$ \\
\hline FEA & $\sqrt{ }$ & $\sqrt{ }$ & & $\sqrt{ }$ \\
\hline
\end{tabular}

or

$$
t(\epsilon)=-\pi \epsilon^{2} ; D_{T} Q=2 \nabla \tilde{u}_{0} \cdot \nabla \tilde{p}_{0}-f \tilde{p}_{0}, \text { when } g=0 .
$$

Here, $g$ represents the external loadings prescribed on boundary $\partial \omega$. We call this the TSA-Circle result for short.

Similarly, for an ellipse with semi-axes $a$ and $b$ parallel to the main axes of the coordinate system, we have [2],

$$
Q(u)=Q\left(u_{0}\right)+a b \pi\left(\nabla \tilde{u}_{0}^{T} T_{\omega} \nabla \tilde{p}_{0}-f \tilde{p}_{0}\right),
$$

where $T_{\omega}$ is defined as

$$
T_{\omega}=\left(\begin{array}{cc}
1+a / b & 0 \\
0 & 1+b / a
\end{array}\right)
$$

We call this the TSA-Ellipse result for short.

(3) Feature sensitivity analysis [27]. Feature sensitivity describes the first order change of a quantity of interest when an arbitrarily-shaped small hole free of external loadings is created within a geometry. The corresponding computation is:

$$
Q(u)=Q\left(u_{0}\right)+\int_{\partial \omega}(V \cdot n)\left(F_{1}+F_{2}\right) d s
$$

where

$$
\begin{gathered}
F_{1}=\frac{f \tilde{p}_{0}-\left(\nabla \tilde{u}_{0}+\nabla u_{E}\right) \cdot\left(\nabla \tilde{p}_{0}+\nabla p_{E}\right)}{2}, \\
F_{2}=f p_{E} / 3, \quad V=P-P_{C},
\end{gathered}
$$

and $u_{E}, p_{E}$ are the corresponding prime and adjoint exterior solutions defined over geometry $R^{n}-\omega, n=2,3$ taking the boundary conditions as those of the solution $u_{0}$ over feature boundary $\partial \omega$.

Estimating modification sensitivity using the different approaches listed above involves solving different PDE problems, for example, over the defeatured model $\Omega$, the fully-featured model $\Omega-\omega$, or the exterior geometry $R^{2}-\omega$, and involves volume or boundary integrations for value evaluations. The requirements are summarized in Table 1.

Basically, solving the field problem over the fullyfeatured model using FE analysis is most time consuming. This is followed in sequence by solving the field problems for the defeatured model, and for the exterior geometry. Solving the prime and adjoint problems using FE methods involves the same stiffness matrix, and solving both is not much more work than solving just one of them using the direct triangular decomposition method. Integration is generally much cheaper than FE analysis, and boundary 
integration is generally cheaper than volume integration. As can be seen from the summary in Table 1, the efficiencies of these related methods are, in increasing order, TSA, our approach, FSA and direct FEA.

We now consider the accuracy of each method. Both TSA and FSA use pointwise values to approximate field values within a suppressed feature based on Eq. (25). This is in contrast to our modification sensitivity estimate, where prime and adjoint solutions $u_{0}, p_{0}$ are used directly without any approximation, and thus it is expected to be more accurate than TSA and FSA particularly for features of large sizes. On the other hand, as we discard the terms involving $\varepsilon_{0}$ in Eq. (22), our method may in principle be less accurate than TSA or FSA for features of sufficiently small size. However, the goal of our paper is to be able to handle features of relatively large size; our experimental results in the next Section demonstrate that this potential issue is not a problem in practice. Finally, we note that FSA is typically more effective than TSA for arbitrarily-shaped features at the cost of additional computations of an auxiliary exterior problem, but both are equally accurate with TSA-Circle for circular features or with TSA-Ellipse for elliptic features.

Our experimental results in the next Section agree with these analyses on efficiency and accuracy. Note all the proposed modification sensitivity analysis is applicable to arbitrary-shape negative features either in the model's interior or along its boundary, while the TSA or FSA is generally only application for negatives features of sufficiently small sizes and far away from the outer boundary.

\section{Numerical experimental results}

Our framework for estimating modification sensitivity has been implemented on a $2.8 \mathrm{GHz}$ dual quad-core processor with 4GB RAM using COMSOL [1], a commercial finite element based CAD/CAE system. We both demonstrate the efficiency and accuracy of our approach, and provide comparisons with those obtained using FEA, TSA and FSA. The Poisson equation described in Section 5 is taken as a concrete example problem for analysis.

In all examples, unless otherwise stated, the quantity of interest is defined as the average of the solution $u$ over a local region $S \in \Omega-\omega$ :

$$
Q(u)=\int_{S} u d \Omega / \int_{S} d \Omega .
$$

Here, when considering accuracy, we use effectivity to assess results. Effectivity of a sensitivity estimator is usually measured in terms of effectivity index, which can be defined as the ratio between the estimated sensitivity $e$ and the exact value $E$, that is

$$
I=e / E
$$

When using a global energy norm, effectivity indices between 0.5 and 2.0 are often characterized as being reasonable values to achieve in the error estimation literature.

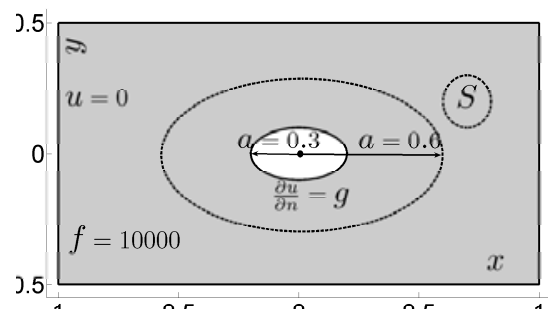

Fig. 4. Removing an internal elliptical hole from a rectangular block.

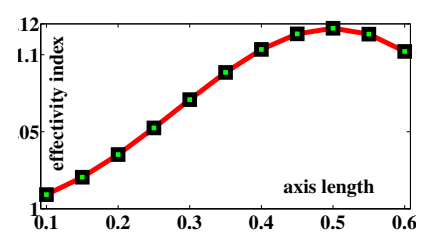

(a) Effectivity index

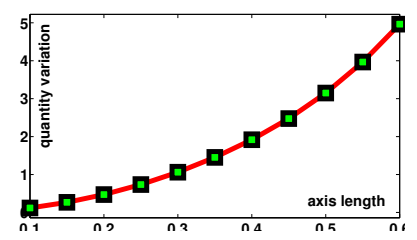

(b) Variation of solution in $S$ as ratio $\left(Q(u)-Q\left(u_{0}\right)\right) / Q\left(u_{0}\right)$
Fig. 5. Effectivity with varying internal feature size in Fig. 4.

However, for goal-oriented error estimation of the kind considered in this paper, it is generally very difficult or expensive to obtain highly accurate error estimates [23]. In practice, effectivity indices up to 10 can still be useful [14]. We leave the reader to form their own judgement.

\subsection{Effectivity testing}

We first consider our method's effectivity under changes of shape of a design feature, and of the boundary conditions prescribed over its boundary.

In the first simple 2D example, the model is an axisaligned rectangle centred at $(0,0)$, containing a single feature, an internal elliptic hole $\omega$ (see Fig. 4). The hole is also centered at $(0,0)$ and has semi-axes of $a$ and $a / 2$ for various values of $a$. The local circular region of interest within which we wish to estimate the average solution is centered at $(0.7,0.2)$ and has radius 0.1 . The corresponding parameters of the Poisson equation in Eq. (24) are set as: $f=1$, $u=0$ along the outer boundary, and $\partial u / \partial n=g$. The effectivity of the modification sensitivity estimator was tested under variations of the features's sizes $a$, locations and the external loadings $g$ as will be explained later. The quantity of interest is defined via Eq. (28).

The modification sensitivity was computed using the proposed method for varying internal feature sizes, locations, and boundary conditions. In the first test, $a$ was changing from 0.1 to 0.6 . The resulting effectivity indices are plotted in Fig. 5(a); they range between 1.0 and 1.12, demonstrating that the method is highly effective in modification sensitivity estimation for various feature sizes.

Note that when $a=0.6$, the internal feature $\omega$ (plotted in dashed line in Fig. 4) is large, occupying over a quarter of the area of the rectangular block, dominating it. This dominance of $\omega$ can be observed in Fig. 5(b), where $I_{Q}=$ $\left(Q(u)-Q\left(u_{0}\right)\right) / Q\left(u_{0}\right)$ is plotted with variation of $a$ to show relative variation in the solution. The change to the average 


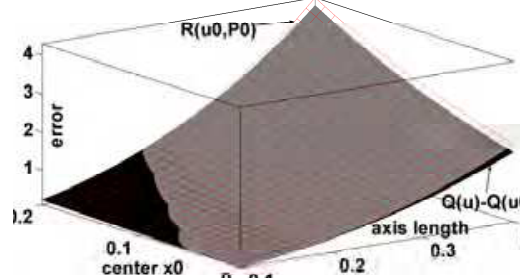

(a) Comparison between $R\left(u_{0} ; p_{0}\right)$ and $Q(u)-Q\left(u_{0}\right)$

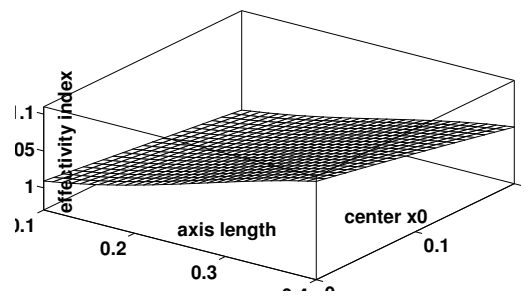

(b) Effectivity index surface

Fig. 6. Performance under variations of the internal feature size and center location for the example in Fig. 4.

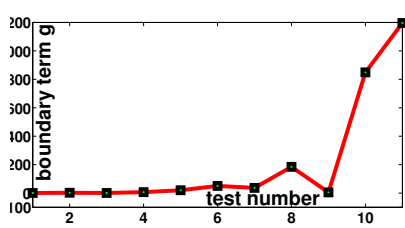

(a) Value of $g$

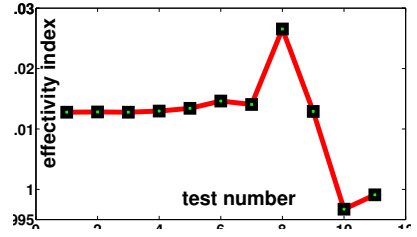

(b) Effectivity index
Fig. 7. Performance under variations of Neumann boundary term $g$ on the internal feature for the example in Fig. 4.

values in $S$ caused by including $\omega$ can be as much as a factor of five compared to the value computed from the defeatured model. Such cases are not atypical of real design problems, and are not handled well by previous approaches.

Our method's effectivity was also tested under variations of both the elliptic feature's size and center: $a$ was varied from 0.1 to 0.6 , and the $x$-coordinate of the center was varied from 0 to 0.2 , leaving the $y$-coordinate unchanged. A comparison between the estimated sensitivity and the exact value $Q(u)-Q\left(u_{0}\right)$ was plotted in Fig. 6(a), and the effectivity index surface was also plotted in Fig. 6(b). The values of the indices are all around 1.0, again showing the algorithm to be highly effective under these variations.

The proposed method can also handle the case when a non-homogeneous Neumann condition is prescribed on the internal feature $\omega$. For the example in Fig. 4, the Neumann boundary term $g$ was randomly set to $2^{i}(i-1) r$ for a random number $0<r<1$ and test number $i=1,2, \ldots, 11$. The applied $g$ values and corresponding effectivity indices are shown in Figures 7. Again, our algorithm is shown to be highly effective.

\subsection{Comparison with topological sensitivity and feature sensitivity}

We next compare the effectivity and efficiency of our method with direct FEA, TSA and FSA. The direct FE

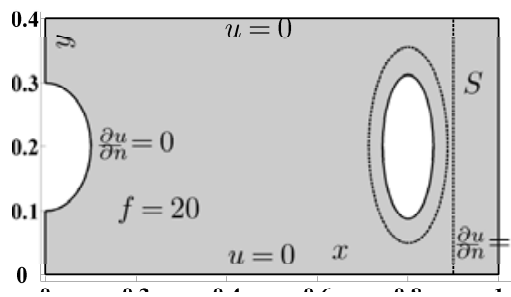

Fig. 8. Example used to compare methods, from [27].

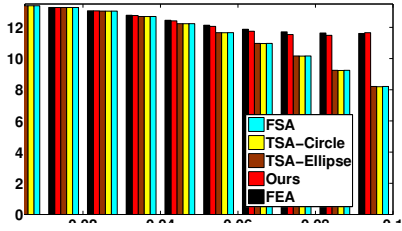

(a) Local quantity of interest: average of $u$ over $S$

Fig. 9. Modification sensitivity estimates using FEA, TSA, FSA and our method for the model in Fig. 8.

analysis results were taken as ground truth for the other approaches. TSA testing considered two different strategies: Eq. (26) for a circular hole (TSA-Circle), and Eq. (27) for an elliptic hole (TSA-Ellipse).

This test used the example in Fig. 8 of an internal elliptic hole $\omega$ with semimajor axis and semiminor axis respectively $a$ and $a / 2$; values of $a$ are explained shortly. This example was taken from [27] with slight modifications: for the Poisson equation as defined in Eq. (24), the source term was given value $f=20$, instead of 1 , to cause bigger values of modification sensitivity. Outer boundary conditions are depicted in Fig. 8; the boundary for the internal elliptic hole was set free. When applying TSA-Circle, a spherical hole with the same cross-section was used as the internal feature instead of this elliptic feature. The quantity of interest is defined via Eq. (28).

In this example, the semi-major axis $a$ was varied between 0.05 and 0.095 . The computed quantities of interest and the effectivity indices for the results obtained by different approaches are summarized and compared in Figs. 9 and 10. The estimated quantity obtained using each approach was defined as $Q\left(u_{0}\right)+e$ for the solution $u_{0}$ over the defeatured model, plus the estimated sensitivity $e$. The bar plots in Fig. 9 show values of the local quantity of interest and the effectivity index obtained by each approach for each test case; the curve plots in Fig. 10 demonstrate their global variation.

Fig. 9(a) shows that the estimated quantity using our approach was always very close to the benchmark results computed using direct FE analysis, for all values of $a$. FSA, TSA-Circle and TSA-Ellipse, however, behave differentlyas the value of $a$ increases, the estimated quantities drastically move away from the benchmark value. Overall variations of the quantities can easily be seen in Figs. 10(a),(b).

We note that the estimates obtained with TSA-Ellipse and FSA always agreed over the whole range of $a$. The former, however, is cheaper to compute as it does not involve 


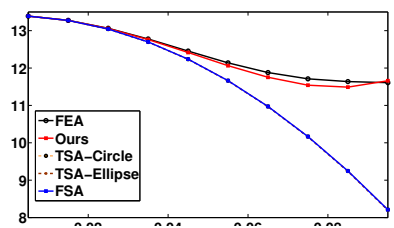

(a) Local quantity of interest: average $u$ over $S$

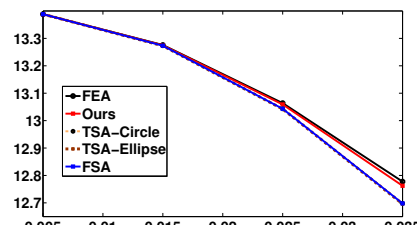

(b) Close-up of local quantity of interest: average of $u$ over $S$

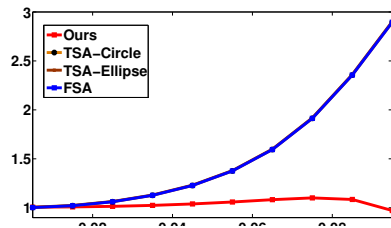

(c) Effectivity index

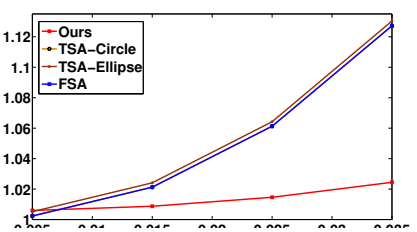

(d) Close-up of effectivity index

Fig. 10. Modification sensitivity estimates using FEA, TSA, FSA and our method for the model in Fig. 8.

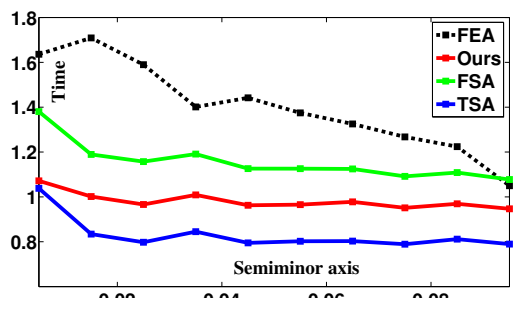

Fig. 11. Time taken by FEA, TSA, FSA and our method for the model in Fig. 8.

solving other auxiliary exterior problems. They are slightly closer to the benchmark FEA results than estimates using TSA-Circle. (Outcomes reported in [27] only compare their approach to TSA-Circle without considering the more accurate TSA-Ellipse).

The corresponding effectivity indices were also compared in Fig. 9(b). Over the whole range of $a$, the effectivity of our estimate was always around 1 . This was in contrast to results of other approaches, which ranged from 1 to 3 . Overall variations of the effectivity indices can easily be seen in Figs. 10(c),(d). This further demonstrates the superior accuracy of our method in estimating modification sensitivity, even for large design changes, unlike previous methods.

In detail (see Figs. 10(b),(d)), when the feature size was relatively small, with a ranging from 0.005 to 0.035 , the computed quantity of interest was very close to the benchmark FEA results for all approaches. In a very small of range of parameter $a$ around 0.005, TSA-Ellipse and FSA perform slightly better than our approach, which can be explained by the fact that we have discarded the term $\bar{h}_{\partial \omega}\left(u_{0}, \varepsilon_{0}\right)-h_{\partial \omega}\left(u_{0}, \varepsilon_{0}\right)$ in Eq. (21). These results demonstrate the minor effects on sensitivity estimation of doing so.

Computation times for this problem, for each method, are given in Fig. 11. As can be seen, the topological sensitivity method was quickest, then our method, then feature sensitivity, and finally direct FE analysis. This is consistent with our discussion in Section 5.

\subsection{D example}

We now consider how our method performed on a somewhat more realistic $3 \mathrm{D}$ example $\mathcal{M}$ with 11 candidate features labeled $L_{1}, \ldots, L_{11}$, shown in Fig. $2(\mathrm{a}) . L_{10}$ and $L_{11}$ are respectively a blind hole and a blend on the bottom. We note that features typically appear on the boundaries of 3D models, unlike 2D models where they are generally in the interior, which means that approaches like TSA or FSA are typically unsuitable. In comparison, our method can be directly applied to 3D models without modification.

The corresponding parameters of the Poisson equation in Eq. (24) were set as follows: the source term was set to $f=1, u=0$ along the planar regions on the model's left and right sides, and the condition $\partial u / \partial n=-1$ was set over the central large cylindrical region. The quantities of interest $Q_{1}(u), Q_{2}(u)$ defined via Eq. (28) were measured over two different regions $S_{1}$ and $S_{2}$ shown in Fig. 2(a). Denote $\mathcal{M}_{0}=\mathcal{M}+\cup_{i=1}^{11} L_{i}$ as the fully defeatured model for later use.

We aim to simplify the model $\mathcal{M}$ by suppressing certain features from $\mathcal{L}=\left\{L_{1}, \ldots, L_{11}\right\}$ so that changes between the field solutions $u_{0}^{i}$ of the final simplified model $\mathcal{M}_{m}^{i}, i=$ 1,2 and solutions $u$ of the full-featured model $\mathcal{M}$ is under certain sensitivity control.

Thus, the corresponding modification sensitivity was first computed to estimate the effects of removing each single feature $L_{i}, i=1, \ldots, 11$, from model $\mathcal{M}$, that is $e\left(\mathcal{M}, \mathcal{M}+L_{i}\right)$; see its definition in Eq. (4). Due to the reason as explained in Section 6, for these 11 features with little interaction, we instead computed sensitivity $e\left(\mathcal{M}_{0}-L_{i}, \mathcal{M}_{0}\right)$, which is approximately equal to $e\left(\mathcal{M}, \mathcal{M}+L_{i}\right)$ but much cheaper to compute; our experimental results also demonstrate their close approximation, which are not further shown here.

In order to demonstrate the effectivity of the modification sensitivity estimation, comparison between the estimated modification sensitivities with the benchmark sensitivities obtained using direct FE analysis for the local quantities defined over $S_{1}$ and $S_{2}$ are shown in Figs. 12(a)(b); values are recorded as 0 when below $10^{-6}$. The associated effectivity indices for regions $S_{1}$ and $S_{2}$ are plotted in Fig. 13.

According to the estimated modification sensitivity $e\left(\mathcal{M}_{0}-L_{i}, \mathcal{M}_{0}\right)$ for each feature $L_{i}$, features $L_{3}$ and $L_{4}$ have a large influence on the quantity defined over region $S_{1}$, showing that a suitable simplified model for this problem should keep these, but delete other features, resulting in a simplified model $\mathcal{M}_{m}^{1}=\mathcal{M}_{0}-L_{3}-L_{4}$. Similarly, a different simplified model $\mathcal{M}_{m}^{2}=\mathcal{M}_{0}-L_{1}-L_{10}$ is suitable for the quantity defined over region $S_{2}$. Table 2 further compares the two groups of models $\mathcal{M}, \mathcal{M}_{0}, \mathcal{M}_{m}^{1}$, and $\mathcal{M}, \mathcal{M}_{0}, \mathcal{M}_{m}^{2}$ in terms of number of mesh elements, solution time, corresponding quantities and difference of quantities from their exact values. 


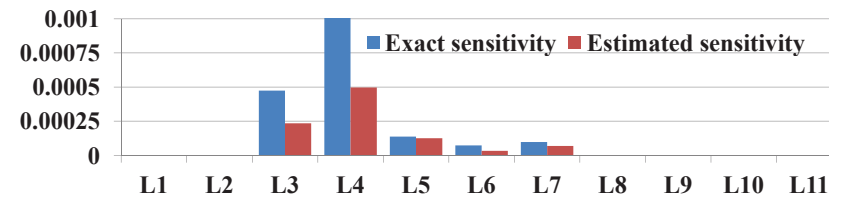

(a) Results for region $S_{1}$

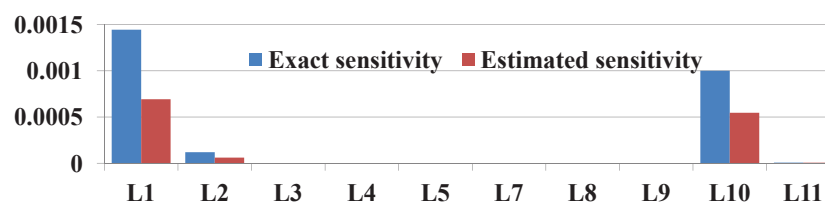

(b) Results for region $S_{2}$

Fig. 12. The estimated modification sensitivity for each feature compared with that obtained using direct FE analysis, for the quantity defined over regions $S_{1}$ and $S_{2}$.

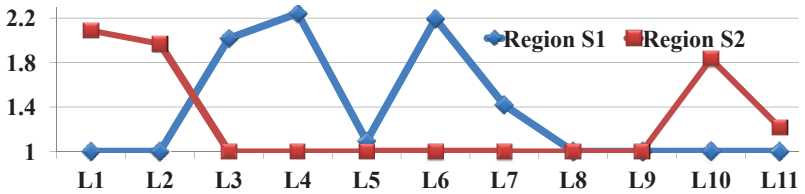

Fig. 13. Effectivity index curves for the quantities of interest defined over regions $S_{1}$ and $S_{2}$.

Table 2

Comparisons of the computational results for the fully-featured model, the fully defeatured model, and the partially defeatured model obtained by our modification sensitivity estimate.

\begin{tabular}{|l|rrr|rrr|}
\hline & \multicolumn{4}{|c|}{$R_{1}$} & \multicolumn{4}{|c|}{$R_{2}$} \\
\hline & $\mathcal{M}$ & $\mathcal{M}_{0}$ & $\mathcal{M}_{m}^{1}$ & $\mathcal{M}$ & $\mathcal{M}_{0}$ & $\mathcal{M}_{m}^{2}$ \\
\hline Mesh Elements & 85132 & 4512 & 20762 & 85132 & 4512 & 27134 \\
\hline Running time $(\mathrm{s})$ & 5.90 & 0.26 & 1.232 & 5.90 & 0.26 & 1.732 \\
\hline Quantity $\left(\times 10^{-6}\right)$ & 4.01 & 3.80 & 3.99 & 4.02 & 3.69 & 3.99 \\
\hline Quantity difference $\left(\times 10^{-6}\right)$ & 0 & 0.21 & 0.02 & 0 & 0.33 & 0.03 \\
\hline
\end{tabular}

As can be observed from the results in Table 2, using the fully-featured model $\mathcal{M}$ is most time consuming; it may even not be affordable for large and complex geometries. Simply using the fully defeatured model $\mathcal{M}_{0}$ significantly reduces the computational time but also leads to large analysis errors. As a trade-off, by keeping certain important features directed by the estimated modification sensitivities, a partially defeatured model $\mathcal{M}_{m}^{1}$ or $\mathcal{M}_{m}^{2}$ of intermediate complexity was obtained which both reduces the analysis complexity, and simultaneously maintains high analysis fidelity. The computational time for solving the analysis problem defined over models $\mathcal{M}, \mathcal{M}_{0}$ and $\mathcal{M}_{m}^{1}, \mathcal{M}_{m}^{2}$ (plus time of modification sensitivity estimations to guide their generations) was also compared in Fig. 14.

Note clearly that the final simplified model $\mathcal{M}_{m}^{1}$ or $\mathcal{M}_{m}^{2}$ was obtained using prime and adjoint solutions defined over the fully defeatured model $\mathcal{M}_{0}$, which only needs to be computed once for all features, and for all regions containing quantities of interest such as $S_{1}$ and $S_{2}$. This saves much computational time.

This example also shows that different local quantities of interest may require different simplified models to main-

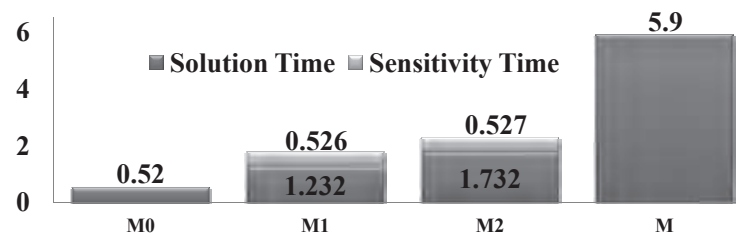

Fig. 14. Computational time in solving the analysis problem over the full-featured model $M$, the fully defeatured model $M_{0}$ and the partially defeatured model $M_{m}^{1}$ and $M_{m}^{2}$ (plus time of modification sensitivity estimations).

tain certain analysis fidelity. Solely using geometry-based simplification criteria is an unsuitable approach.

\section{Conclusions and future work}

As can be observed from the experimental results, our novel method for estimating modification sensitivity is both accurate and efficient even for large negative features. Such cases cannot be well handled by previous approaches such as topological sensitivity analysis or feature sensitivity analysis, which were mainly intended for design optimization, and focus on relatively small changes.

Our method has similar computational efficiency to topological sensitivity analysis, in that only prime and adjoint solutions of the defeatured model need to be computed for all non-interacting features, and it is less computationally expensive than the approach of feature sensitivity analysis, as the latter requires certain additional solutions to auxiliary exterior problems for each feature.

Moreover, due to use of the adjoint formulation, the error can be efficiently estimated with little extra effort when a feature changes in shape, size, location, or boundary conditions. Since repeated design changes are required in simulation-based design, and also as CAD models typically contains many different features at different locations and of different shapes, this will save much computational cost.

However, the approach here is limited to negative features with Neumann boundary conditions, where the fullyfeatured model is contained within the defeatured model. A companion paper [20] considers its extension to positive features. The key issue involved in this extension is how to derive suitable boundary conditions outside the defeatured model so that the conversion from modification sensitivity to modeling error is still possible. We are also exploring applying Nitsche's method to handle cases of general features with prescribed Dirichlet boundary conditions.

\section{Appendix A. Modification sensitivity estimators}

Using the notation in Section 4.4, we have

$$
F_{0}(v)=\left.(f, v)\right|_{\Omega}, F(v)=(f, v)_{\Omega-\omega}
$$

and

$$
F_{0}(v)=F(v)+\left.(f, v)\right|_{\omega} .
$$

From the definition of $N(u, v)$, we have 


$$
\begin{aligned}
N(u, v) & =\left.(L u, v)\right|_{\Omega-\omega} \\
& =\bar{H}_{\partial \Omega}(u, v)+\bar{h}_{\partial \omega}(u, v)-\left.L^{*}(u, v)\right|_{\Omega-\omega} .
\end{aligned}
$$

By observing that solutions $u$ and $u_{0}$ share the same boundary conditions over $\partial \Omega$, we also have

$$
N_{0}\left(u_{0} ; v\right)=\left.\left(L u_{0}, v\right)\right|_{\Omega}=\bar{H}_{\partial \Omega}\left(u_{0}, v\right)-\left.L^{*}\left(u_{0}, v\right)\right|_{\Omega} .
$$

Taking into account Eq. $(20)$, the residual $R\left(u_{0} ; v\right)$ defined by Eq. (11) then becomes

$$
\begin{aligned}
& R\left(u_{0} ; v\right)=N\left(u_{0} ; v\right)-F(v)= \\
& \quad \bar{H}_{\partial \Omega}\left(u_{0}, v\right)+\bar{h}_{\partial \omega}\left(u_{0}, v\right)-\left.L^{*}\left(u_{0}, v\right)\right|_{\Omega-\omega}-\left.(f, v)\right|_{\Omega-\omega} .
\end{aligned}
$$

By observing that $H_{\partial \Omega}\left(u_{0}, v\right)=\bar{H}_{\partial \Omega}\left(u_{0}, v\right)$, we get

$$
\begin{aligned}
& \left.L^{*}\left(u_{0}, v\right)\right|_{\Omega-\omega}=H_{\partial \Omega}\left(u_{0}, v\right)+h_{\partial \omega}\left(u_{0}, v\right)-\left.\left(L u_{0}, v\right)\right|_{\Omega-\omega}, \\
& R\left(u_{0} ; v\right)=\bar{h}_{\partial \omega}\left(u_{0}, v\right)-h_{\partial \omega}\left(u_{0}, v\right)+\left.\left(L u_{0}, v\right)\right|_{\Omega-\omega}-\left.(f, v)\right|_{\Omega-\omega} .
\end{aligned}
$$

As $u_{0}$ is a solution to Eq. (2), this ultimately gives

$$
R\left(u_{0} ; v\right)=\bar{h}_{\partial \omega}\left(u_{0}, v\right)-h_{\partial \omega}\left(u_{0}, v\right) .
$$

Replacing $v$ by $p, p_{0}$ or $\varepsilon_{0}=p-p_{0}$ in Eq. (A.1), we have

$$
Q(u)-Q\left(u_{0}\right) \approx \mathcal{R}\left(u_{0} ; p\right)=\bar{h}_{\partial \omega}\left(u_{0}, p\right)-h_{\partial \omega}\left(u_{0}, p\right) .
$$

\section{Acknowledgements}

We thank the reviewers for their comments, which have helped to improve the paper. This work described in this paper was partially supported by the NSF of China (No.60736019), the National Basic Research Program of China (2011CB302400), the 863 High Technology Plan of China (2008AA04Z115) and the Fundamental Research Funds for the Central Universities (1A5000*172210101[43]).

\section{References}

[1] Comsol multiphysics 3.5a. http://www.comsol.com., 2007.

[2] S. Amstutz. Sensitivity analysis with respect to a local perturbation of the material property. Asymptotic Analysis, 49:87-108, 2006.

[3] R. Becker and R. Rannacher. A feed-back approach to error control in finite element methods: basic analysis and examples. East-West Journal of Numerical Mathematics, 4:237-264, 1996.

[4] R. Becker and R. Rannacher. An optimal control approach to a posteriori error estimation in finite element methods. Acta Numerica, 10:1-102, 2001.

[5] P. Bettess. Infinite Elements. Penshaw Press: Sunderfand, 1992.

[6] KK Choi and NH Kim. Structural Sensitivity Analysis and Optimization I: Linear Systems. Springer, New York, 2005.

[7] J. Cnossen. Goal-oriented modelling-error estimation for hierarchical models of a different type. PhD thesis, Faculty of Aerospace Engineering, Delft University of Technology, 2007.

[8] R. Cook, D. Malkus, M. Plesha, and R. Witt. Concepts and Appl. of Finite Element Analysis. John Wiley \& Sons, 2002.

[9] A. Embar, J. Dolbow, and I. Harari. Imposing Dirichlet boundary conditions with Nitsche's method and spline-based finite elements. International Journal for Numerical Methods in Engineering, 83:877-898, 2010.
[10] HA Eschenauer and N. Olhoff. Topology optimization of continuum structures: a review. Applied Mechanics Review, 54(4):331-390, 2001.

[11] RA Feij, AA Novotny, E. Taroco, and C. Padra. The topological derivative for the Poisson's problem. Mathematical Models and Methods in Applied Sciences, 13(12):1825-1844, 2003.

[12] S. Fernandez-Mendez and A. Huerta. Imposing essential boundary conditions in mesh-free methods. Computer Methods in Applied Mechanics and Engineering, 193:1257-1275, 2004.

[13] R. Ferrandes, P. Marin, J. Leon, and F. Giannini. A posteriori evaluation of simplification details for finite element model preparation. Computers and Structures, 87(1-2):73-80, 2009.

[14] M. Giles and E. Suli. An introduction to a posteriori error analysis and adjoints. Technical report, NASA Ames Research Center, CA, and The von Karman Institute for Fluid Dynamics, Belgium, 2010.

[15] S. Gopalakrishnan and K. Suresh. Estimating the impact of large design changes on field problems. In Proceedings of the 2007 ACM symposium on Solid and physical modeling, pages 205-215, New York, NY, USA, 2007. ACM.

[16] T. Gratsch and K. Bathe. A posteriori error estimation techniques in practical finite element analysis. Computers \& Structures, 83(4-5):235-265, 2005.

[17] D. Kelly, J. De, S. Gago, O. Zienkiewicz, and I. Babuska. A posteriori error analysis and adaptive processes in the finite element method: Part I - error analysis. Int. J. Numer. Meth. Engng, 19(11):1593C1619, 1983.

[18] U. Kirsch. Reanalysis and sensitivity reanalysis by combined approximations. Struct Multidisc. Optim., 40(1-6):1-15, 2010.

[19] K. Lee, C. Armstrong, M. Price, and J. Lamont. A small feature suppression/unsuppression system for preparing b-rep models for analysis. In Proc. of the ACM Symposium on Solid and Physical Modeling, pages 113-124, Cambridge, MA, 2005.

[20] Ming Li and Shuming Gao. Estimating defeaturing-induced engineering analysis error for arbitrary 3D features. Submitted.

[21] S. Nazarov and J. Sokolowski. Asymptotic analysis of shape functionals. Journal de Mathématiques Pures et Appliqués, 82(2):125-196, 2003.

[22] J. Oden and S. Prudhomme. Estimation of modeling error in computational mechanics. Journal of Computational Physics, 182:496-515, 2002.

[23] S. Prudhomme, J. Oden, T. Westermann, J. Bass, and M. Botkin. Practical methods for a posteriori error estimation in engineering applications. Int. J. Numer. Meth. Engng, 56:1193$1224,2003$.

[24] Y. Saad. Iterative methods for sparse linear systems. Halstead Press, 2003.

[25] J. Sokolowski and A. Zochowski. On topological derivative in shape optimization. SIAM Journal of Control Optimization, 37(4):1251-1272, 1999.

[26] A. Thakur, A. Banerjee, and S. Gupta. A survey of CAD model simplification techniques for physics-based simulation applications. Computer-Aided Design, 41(2):65-80, 2009.

[27] I. Turevsky, S. Gopalakrishnan, and K. Suresh. Defeaturing: A posteriori error analysis via feature sensitivity. Int. J. Numer. Meth. Engng, 76(9):1379-1401, 2008.

[28] I. Turevsky, S. Gopalakrishnan, and K. Suresh. An efficient numerical method for computing the topological sensitivity of arbitrary-shaped features in plate bending. Int. J. Numer. Meth. Engng, 79(13):1683-1702, 2009.

[29] DR White, S. Saigal, and SJ Owen. Meshing complexity of single part cad models. In Proceedings of the 12th International Meshing Roundtable Conference, Santa Fe, New Mexico, U.S.A., 2003. 